\title{
REGULARITY PROPERTIES OF OPTIMAL CONTROLS FOR PROBLEMS WITH TIME-VARYING STATE AND CONTROL CONSTRAINTS
}

\author{
Ilya A. Shvartsman \\ Richard B. Vinter \\ Dept. of Electrical and Electronic Engineering, \\ Imperial College of Science, Technology and Medicine, \\ Exhibition Road, London, UK, SW7-2AZ.
}

\begin{abstract}
In this paper we report new results on the regularity of optimal controls for dynamic optimization problems with functional inequality state constraints, a convex time-dependent control constraint and a coercive cost function. Recently it has been shown that the linear independence condition on active state constraints, present in the earlier literature, can be replaced by a less restrictive, positive linear independence condition, that requires linear independence merely with respect to non-negative weighting parameters, provided the control constraint set is independent of the time variable. We show that, if the control constraint set, regarded as a time dependent multifunction, is merely Lipschitz continuous with respect to the time variable, then optimal controls can fail to be Lipschitz continuous. In these circumstances, however, a weaker Hölder continuity-like regularity property can be established. On the other hand, Lipschitz continuity of optimal controls is guaranteed for time varying control sets under a positive linear independence hypothesis, when the control constraint sets are described, at each time, by a finite collection of functional inequalities.
\end{abstract}

Key Words. Optimal control, Lipschitz controls, normal necessary conditions.

AMS Subject Classification (2000). Primary: 49N60, 49J30. Secondary: 49J52.

\section{Introduction}

Consider the following optimal control problem with pathwise state and control constraints and an endpoint constraint:

$$
(\mathcal{P})\left\{\begin{array}{l}
\text { Minimize } g(x(S), x(T))+\int_{S}^{T} L(t, x(t), u(t)) d t \\
\text { over } x \in W^{1,1} \text { and measurable } u:[S, T] \rightarrow \mathbb{R}^{m} \\
\text { satisfying } \\
\dot{x}(t)=f(t, x(t))+G(t, x(t)) u(t) \text { a.e. } t \in[S, T] \\
h_{j}(t, x(t)) \leq 0 \text { for a.e. } t \in[S, T], j=1, \ldots, r, \\
u(t) \in U(t) \text { a.e. } t \in[S, T] \\
(x(S), x(T)) \in C .
\end{array}\right.
$$

The data for this problem comprise an interval $[S, T]$, functions $L:[S, T] \times \mathbb{R}^{n} \times \mathbb{R}^{m} \rightarrow \mathbb{R}$, $f:[S, T] \times \mathbb{R}^{n} \rightarrow \mathbb{R}^{n}, G:[S, T] \times \mathbb{R}^{n} \rightarrow \mathbb{R}^{n \times m}, h_{j}: \mathbb{R} \times \mathbb{R}^{n} \rightarrow \mathbb{R}$ for $j=1, \ldots, r$, a closed set $C \subset \mathbb{R}^{n} \times \mathbb{R}^{n}$ and a multifunction $U:[S, T] \rightarrow \mathbb{R}^{m}$.

For much of the analysis, we shall assume $U(t)$ has representation:

$$
U(t)=\bigcap_{j=1}^{l}\left\{u \mid d_{j}(t, u) \leq 0\right\} .
$$


Here $d_{j}:[S, T] \times \mathbb{R}^{m} \rightarrow \mathbb{R}$, for $j=1, \ldots, l$ are given functions. We allow the cases $r=0$ (no state constraints) and $l=0$ (no control constraints).

A control function is a measurable function $u:[S, T] \rightarrow \mathbb{R}^{m}$ such that $u(t) \in U(t)$ for a.e. $t \in[S, T]$. A process $(x, u)$ comprises a control function $u$ and a $W^{1,1}$ function $x$ satisfying the constraints of $(\mathcal{P})$. We say the process $(\bar{x}, \bar{u})$ is a minimizer if it achieves the minimum. In this case, $\bar{u}$ and $\bar{x}$ are referred to as an optimal control and an optimal state trajectory (corresponding to $\bar{u})$, respectively. Fix an optimal process $(\bar{x}, \bar{u})$.

In this paper we report new sets of conditions, under which optimal controls have Lipschitz or Hölder continuity-like regularity propoerties. The significance of such conditions are discussed in [5]. We mention, in particular, that prior knowledge of the regularity properties of optimal controls ('bounded slope') influences the choice of the most effective approximation scheme for numerical solution of optimal control problems and the rates of convergence that can be achieved.

A key advance in the quest for conditions assuring Lipschitz continuity of optimal controls in the presence of both state and control functional inequality constraints was provided by Hager's 1979 paper [6] in which Lipschitz continuity of optimal controls (for linear-convex problems) was established under hypotheses that included the constraint qualification:

(LI) There exists $\gamma>0$ such that for all collections of numbers $\alpha_{1}, \ldots, \alpha_{r}$ and $\beta_{1}, \ldots, \beta_{l}$ and for each $t \in[S, T]$ we have

$$
\left|G^{T}(t, \bar{x}(t)) \sum_{j} \alpha_{j} \nabla_{x} h_{j}(t, \bar{x}(t))+\sum_{j^{\prime}} \beta_{j^{\prime}} \nabla_{u} d_{j^{\prime}}(t, \bar{u}(t))\right| \geq \gamma\left(\sum_{j}\left|\alpha_{j}\right|^{2}+\sum_{j^{\prime}} \beta_{j^{\prime}}^{2}\right)^{1 / 2},
$$

where each $\nabla_{x} h_{j}, \nabla_{u} d_{j^{\prime}}$ is interpreted as a column vector. Summations are taken over values of the index $j$, for which the relevant constraints are active. ('linear independence of active state constraints').

Malanowski [7] extended Hager's analysis, establishing Lipschitz continuity of optimal controls under less restrictive conditions, that allow dynamics nonlinear with respect to the state variable and a cost integrand which is, possibly, nonconvex with respect to the state variable. Alternative proofs and additional regularity properties of optimal controls under certain circumstances ('piecewise analyticity') where later proved by Dontchev et al. [3], [4].

Recently, Galbraith and Vinter [5] considered problems involving a pathwise control constraint

$$
u(t) \in U(t) \text { a.e. } t \in[S, T],
$$

where $U(\cdot)$ is a multifunction on $[S, T]$, taking values closed, convex sets, not necessarily expressible in terms of a finite collection of inequality constraints. Lipschitz continuity was established under hypotheses that included

$$
U(t) \text { is independent of } t \text { (write it } U \text { ) }
$$

and also a modified constraint qualification (LI) in which a version of inequality (1.2) was required to be satisfied, merely for non-negative numbers $\alpha$ 's ('positive linear independence with respect to the state constraints'). Specifically, it was required that

There exists $\varepsilon>0$ such that for every collection of positive numbers $\alpha_{1}, \ldots, \alpha_{r}$ and at any time $t \in[S, T]$ we have

$$
\sum_{j} \alpha_{j} G^{T}(t, \bar{x}(t)) \nabla_{x} h_{j}(t, \bar{x}(t)) \notin \operatorname{span} \bigcup_{u \in(\bar{u}(t)+\varepsilon B) \cap U} N_{U}(u) .
$$

(In [5], the condition was stated with $\varepsilon=0$, but validity of the analysis requires formulation of the condition in terms of some positive $\varepsilon$. ) 
The weaker positive-type linear independence hypotheses had previously arisen as conditions for normality of multiplier sets but not, apparently, in regularity analysis.

In this paper we examine regularity properties of optimal controls, in circumstances when the control constraint set $U(t)$ is time varying. Here, one might expect that optimal controls are Lipschitz continuous, when we assume

$t \rightarrow U(t)$ is Lipschitz continuous with respect to the Hausdorff metric.

Concerning this conjecture, we provide a counter-example in which conditions of this nature are satisfied, yet the optimal control is merely Hölder continuous with index $1 / 2$. We show that, consistent with the counter-example, optimal controls have a Hölder continuity-like property with index $1 / 2$, for a wide class of problems involving Lipschitz continuous time dependent control constraint sets, in the above sense. Hölder continuity (with index 1/2) has earlier featured in sensitivity analysis for parameterized optimization problems with an implicit constraint [2], [1].

Our results assert, furthermore, that for the time varying case, optimal controls are Lipschitz continuous if we revert to a problem formulation, in which the control constraint is described in terms of a finite number of smooth functional inequalities (1.1) and when we merely require condition (LI) above to be satisfied for non-negative $\alpha$ 's.

The conditions for Lipschitz/Hölder continuity of optimal controls are obtained by means of a detailed analysis of the implications of the nonsmooth Maximum Principle. A key step is to consider the properties of trajectory sub-arcs with the property that all state constraints active at some intermediate time are active also at the end-times; the significance of such sub-arcs for regularity investigations was earlier emphasized by Hager ([6], Thm. 2.1). The analysis greatly simplifies if the control constraints are absent, the cost is quadratic in the $u$ variable and there is only one state constraint. (See ([11], Ch.11).)

Note that research efforts following on from Hager's 1979 paper have been directed, in part, towards assembling a set of hypotheses assuring regularity of minimizers, uniqueness of multipliers and smooth dependence on parameters, and constructing a framework for numerical solution techniques involving 'dual' concepts. The present paper is of narrower focus, concentrating exclusively on conditions for regularity of optimal controls. If this alone is our goal, then the linear independence hypotheses of the earlier literature can be relaxed to positive linear independence. Note however that, under this positive linear independence hypothesis, the state constraint multipliers may fail to be unique.

Finally, some notation. $|$.$| denotes the Euclidean norm. The closed unit ball in Euclidean$ space is written $\mathbb{B} . C^{\oplus}(S, T)$ denotes the space of non-negative Borel measures on the Borel subsets of $[S, T]$. For a given subset $A \subset \mathbb{R}^{k}, \Psi_{A}$ denotes the indicator function:

$$
\Psi_{A}(y)= \begin{cases}0 & \text { if } y \in A \\ +\infty & \text { otherwise }\end{cases}
$$

We make use of a few standard constructs from nonsmooth analysis (see, for example, [8] and [10] for full details). The normal cone and the limiting subgradient are defined as follows.

Definition 1.1 Take a closed set $C \subset \mathbb{R}^{n}$ and a point $\bar{x} \in C$. We say that $y \in \mathbb{R}^{n}$ is a normal to $C$ at $\bar{x}$ if there exist $y_{i} \rightarrow y$ and $x_{i} \rightarrow \bar{x}$ (in $C$ ) such that for all $i$,

$$
\left\langle y_{i}, x-x_{i}\right\rangle \leq o\left(\left|x-x_{i}\right|\right)
$$

for all $x \in C$. The normal cone to $C$ at $\bar{x}$, written $N_{C}(\bar{x})$, is the set of all normals to $C$ at $\bar{x}$. (It is also referred to as the limiting normal cone.) 
Given a lower semicontinuous (lsc) function $f: \mathbb{R}^{n} \rightarrow \overline{\mathbb{R}}$, we denote by $\partial f(\bar{x})$ the subgradient of $f$ at $\bar{x}$ (also known as the limiting subgradient), defined as

$$
\partial f(\bar{x}):=\left\{y:(y,-1) \in N_{\text {epi } f}(\bar{x}, f(\bar{x}))\right\},
$$

in which epi $f$ denotes the set $\left\{(x, \alpha) \in \mathbb{R}^{n} \times \mathbb{R}: \alpha \geq f(x)\right\}$.

\section{The Maximum Principle and Normality}

Denote by $\mathcal{H}: \mathbb{R} \times \mathbb{R}^{n} \times \mathbb{R}^{n} \times \mathbb{R}^{m} \times \mathbb{R} \rightarrow \mathbb{R}$ the unmaximized Hamiltonian

$$
\mathcal{H}(t, x, p, u, \lambda)=\langle p, f(t, x)+G(t, x) u\rangle-\lambda L(t, x, u) .
$$

Let $(\bar{x}, \bar{u})$ be a minimizing process. Under mild hypotheses, and, in particular, under hypotheses (H1)-(H4) of Section 3, necessary conditions of optimality, known as the (state constrained) Maximum Principle [11], provide the following information about $(\bar{x}, \bar{u})$.

There exist 'multipliers' $p \in W^{1,1}\left([S, T] ; \mathbb{R}^{n}\right), \mu_{j} \in C^{\oplus}(S, T)$ for $j=1, \ldots, r$, and $\lambda \geq 0$ such that, writing

$$
q\left(t^{-}\right)=p(t)+\sum_{j=1}^{r} \int_{[S, t)} \nabla_{x} h_{j}(s, \bar{x}(s)) \mu_{j}(d s),
$$

we have

$$
\begin{gathered}
(p, \mu, \lambda) \neq(0,0,0), \\
-\dot{p}(t) \in \operatorname{co} \partial_{x} \mathcal{H}\left(t, \bar{x}(t), q\left(t^{-}\right), \bar{u}(t), \lambda\right) \text { a.e. } t \in[S, T], \\
\mathcal{H}\left(t, \bar{x}(t), q\left(t^{-}\right), \bar{u}(t), \lambda\right)=\max _{u \in U(t)} \mathcal{H}\left(t, \bar{x}(t), q\left(t^{-}\right), u, \lambda\right) \text { a.e. } t \in[S, T], \\
\operatorname{supp}\left\{\mu_{j}\right\} \subset\left\{t: h_{j}(t, \bar{x}(t))=0\right\} \quad \text { for } j=1, \ldots, r, \\
\left(p(S),-\left[p(T)+\sum_{j=1}^{r} \int_{[S, T]} \nabla_{x} h_{j}(t, \bar{x}(t)) \mu_{j}(d t)\right]\right) \\
\in \lambda \partial g(\bar{x}(S), \bar{x}(T))+N_{C}(\bar{x}(S), \bar{x}(T)) .
\end{gathered}
$$

A process for which these conditions are satisfied is said to be an extremal.

The methodology behind the ensuing analysis is to deduce regularity properties of optimal controls from the conditions of the Maximum Principle. It is inevitable then that some kind of hypothesis on the data for problem $(\mathcal{P})$ is imposed, ensuring that the Maximum Principle supplies useful information about the minimizer $(\bar{x}, \bar{u})$. This hypothesis is normality. If it is possible to satisfy the conditions of the Maximum Principle with a set of multipliers $\left(p, \mu_{1}, \ldots \mu_{r}, \lambda\right)$ in which $\lambda=0$, the Maximum Principle makes no reference to the cost function and degenerates into a relationship between the constraints. 'Normality' means that this kind of degeneracy is excluded.

Definition 2.1 A process $(\bar{x}, \bar{u})$ is said to be a normal extremal if there exist $p \in W^{1,1}\left([S, T] ; \mathbb{R}^{n}\right)$ and $\mu_{j} \in C^{\oplus}(S, T), j=1, \ldots, r$ such that the relationships (2.2)-(2.7) are satisfied with $\lambda=1$.

Verifiable conditions for normality expressible directly in terms of the data can be found, for example, in [5]. 


\section{Conditions for Lipschitz Continuity of Normal Extremals}

In this section, we focus on the case when $U(t)$ has representation (1.1). We shall invoke the following set of hypotheses for $(\mathcal{P})$; reference is made here to the process $(\bar{x}, \bar{u})$ of interest. In the hypotheses, $\Omega \subset[S, T] \times \mathbb{R}^{n}$ is some 'tube' around $\bar{x}$, that is

$$
\Omega=\left\{(t, x) \in[S, T] \times \mathbb{R}^{n}:|x-\bar{x}(t)| \leq \bar{\varepsilon}\right\}
$$

(for some given $\bar{\varepsilon}>0$ ). We denote by $\mathcal{J}(t, x)$ and $\mathcal{T}(t, u)$ the collection of active state and control constraints respectively, that is

$$
\mathcal{J}(t, x)=\left\{j \mid h_{j}(t, x)=0\right\}, \mathcal{T}(t, u)=\left\{j \mid d_{j}(t, u)=0\right\} .
$$

(H1) $G, f, L$ and $l$ are locally Lipschitz continuous functions.

(H2) For $j=1, \ldots, r, h_{j}$ is of class $\mathcal{C}^{1+}$ on $\Omega$, i.e., $h_{j}$ is continuously differentiable with locally Lipschitz continuous gradient.

(H3) $U(\cdot)$ is a closed convex-valued multifunction, Lipschitz continuous with respect to the Hausdorff metric.

(H4) For each $(t, x) \in \Omega$ the function $u \rightarrow L(t, x, u)$ is

a) of class $C^{2}$;

b) strongly convex in the following uniform sense: there exists a constant $\sigma>0$ such that for each $(t, x) \in \Omega$

$$
\begin{aligned}
& L\left(t, x,(1-\lambda) u_{1}+\lambda u_{2}\right) \\
& \quad \leq(1-\lambda) L\left(t, x, u_{1}\right)+\lambda L\left(t, x, u_{2}\right)-\frac{1}{2} \sigma \lambda(1-\lambda)\left|u_{1}-u_{2}\right|^{2}
\end{aligned}
$$

for all $u_{1}, u_{2} \in \mathbb{R}^{m}$ and $\lambda \in(0,1)$. Furthermore, for any compact sets $D \subset \mathbb{R}^{m}, E \in$ $\mathbb{R}^{1+n}$, the function $(t, x) \rightarrow \nabla_{u} L(t, x, u)$ is Lipschitz continuous on $E$ uniformly with respect to $u \in D$.

(H5) $U(\cdot)$ has the representation (1.1). Furthermore, for $j=1, \ldots, l$,

a) the function $u \rightarrow d_{j}(t, u)$ is of class $C^{2}$ and convex for all $t \in[S, T]$;

b) the functions $t \rightarrow d_{j}(t, u), t \rightarrow \nabla_{u} d_{j}(t, u)$ are locally Lipschitz continuous.

c) the vectors $\left\{\nabla_{u} d_{j}(t, \bar{u}(t)), j \in \mathcal{T}(t, \bar{u}(t))\right\}$ are linearly independent for all $t \in[S, T]$.

(H6) For the continuous representation of $\bar{u}(\cdot)^{1}$ (write it also $\bar{u}(\cdot)$ ), for any $t \in[S, T]$ such that $\mathcal{J}(t, \bar{x}(t)) \neq \emptyset, \mathcal{T}(t, \bar{u}(t)) \neq \emptyset$, any collection of non-negative numbers $\left\{\alpha_{j}\right\}_{j \in \mathcal{J}(t, \bar{x})}$, and numbers $\left\{\beta_{j}\right\}_{j \in \mathcal{T}(t, \bar{u})}$, not all zero, we have

$$
\sum_{j \in \mathcal{J}(t, \bar{x}(t))} \alpha_{j} G^{T}(t, \bar{x}(t)) \nabla_{x} h_{j}(t, \bar{x}(t))+\sum_{j \in \mathcal{T}(t, \bar{u}(t))} \beta_{j} \nabla_{u} d_{j}(t, \bar{u}(t)) \neq 0
$$

See [2], Chapter 1 and [10] for historical background on the strong convexity hypothesis of (H4)(b). A straightforward analysis provides the following implications of the strong convexity property, which we state without proof.

Lemma 3.1 Assume (H4)(b). Let $\sigma$ be the constant of (H4).

\footnotetext{
${ }^{1}$ It is proved below in Lemma 5.3 that under hypotheses $(\mathrm{H} 1)-(\mathrm{H} 4), \bar{u}(\cdot)$ has a continuous representation.
} 
(i) Fix $(t, x) \in \Omega$. Then the function $u \rightarrow L(t, x, u)-\frac{1}{2} \sigma|u|^{2}$ is convex. If $u \rightarrow L(t, x, u)$ is of class $C^{2}$ then

$$
\nabla_{u u}^{2} L(t, x, u) \geq \sigma I \quad \text { for all } u \in \mathbb{R}^{m},
$$

where $I$ is the identity matrix.

(ii) Fix $(t, x) \in \Omega$. Then

$$
\left\langle y_{2}-y_{1}, u_{2}-u_{1}\right\rangle \geq \sigma\left|u_{2}-u_{1}\right|^{2}
$$

where $y_{2}=\nabla_{u} L\left(t, x, u_{2}\right)$ and $y_{1}=\nabla_{u} L\left(t, x, u_{1}\right)$.

(iii) The function $u \rightarrow L(t, x, u)$ is uniformly coercive, in the sense that there exists a monotone function $\theta:[0, \infty) \rightarrow \mathbb{R}$, such that $\theta(s) / s \rightarrow \infty$ as $s \rightarrow \infty$ and

$$
L(t, x, v)>\theta(|v|) \quad \text { for all }(t, x) \in \Omega \text { and } v \in U(t) .
$$

The stage is now set for statement of conditions for Lipschitz continuity of optimal controls, when the control constraint set is expressible in terms of functional inequalities.

Theorem 3.2 Let $(\bar{x}, \bar{u})$ be a normal extremal to $(\mathcal{P})$ with control constraint given by (1.1). Assume (H1)-(H6). Then $\bar{u}$ is Lipschitz continuous. If $r=0$ (no state constraints), this assertion is valid merely under hypotheses (H1)-(H5).

We remark, once again, that Theorem 3.2 improves on earlier regularity results in $[6],[7]$, by merely invoking a positive linear independence hypothesis regarding the state constraints. It improves also on [5] by allowing a time-varying control constraint set (provided it has representation (1.1)).

\section{Regularity of Optimal Controls for General, Time-Varying Control Constraint Sets.}

We consider again the control problem $(\mathcal{P})$. Now, however, we do not assume the functional inequality representation (1.1) on the control constraint set, but retain the general description:

$$
u(t) \in U(t) \quad \text { a.e. } t \in[S, T] .
$$

In [5] it was shown that optimal controls are Lipschitz continuous, under hypotheses (H1),(H2) and (H4), when condition (1.3) is satisfied and when additionally it is assumed that the control constraint set $U(t)$ is closed, convex and independent of time.

Do optimal controls continue to remain Lipschitz continuous, when we allow the control constraint set to be time-varying?

One might expect that optimal controls are Lipschitz continuous, under hypotheses (H1), (H2) and (H4), when (1.3) is satisfied and when, furthermore, $U(t)$ is Lipschitz continuous with respect to the Hausdorff metric. The following example establishes that this conjecture is false.

\section{Example 4.1}

Consider the following example of $(\mathcal{P})$ in which $T=(\pi / 4)^{2}$ :

$$
\left\{\begin{array}{l}
\text { Minimize } \int_{0}^{T}|u(t)|^{2} d t \\
\text { over } x \in W^{1,1} \text { and measurable } u:[0, T] \rightarrow \mathbb{R}^{2} \\
\text { satisfying } \\
\dot{x}(t)=0, \text { a.e. } t \in[0, T] \\
u(t) \in U(t) \\
(x(0), x(T)) \in\{0\} \times \mathbb{R} .
\end{array}\right.
$$


Here, the state and control variables are of dimension 1 and 2, respectively. The time-varying control constraint set is:

$$
U(t):=\operatorname{co}\{(0, \sec \sqrt{t}),(\sin \sqrt{t}, \cos \sqrt{t}),(1,1)\} .
$$

The data for this example satisfies hypotheses (H1)-(H4). Indeed, (H1),(H2),(H4) are obviously satisfied. It is clear also that $t \rightarrow U(t)$ is locally Lipschitz continuous on $(0, T]$. Furthermore,

$$
d(U(0), U(t))=\max \{(\sec \sqrt{t}-1),(1-\cos \sqrt{t})\}=\sec \sqrt{t}-1 \leq \frac{t}{2}+o(t),
$$

where $o(\cdot): \mathbb{R} \rightarrow \mathbb{R}$ is a function such that $\lim _{t \downarrow 0} \frac{o(t)}{t}=0$. These facts combine to establish Lipschitz continuity of $U(\cdot)$ on the closed interval $[0, T]$. The constraint qualification condition (1.3) of the introduction (or rather a version of it applied to problems with time-dependent control constraint sets, see hypothesis (H6A) below), is vacuously satisfied because there are no state constraints.

Notice that the cost integrand is independent of the state variable and the right endpoint of the state trajectory is unconstrained. It follows that the minimizing control $\bar{u}(\cdot)$ is the pointwise minimizer of the integral over the control constraint sets $U(t), 0 \leq t \leq T$, namely

$$
\bar{u}(t)=(\sin \sqrt{t}, \cos \sqrt{t}) .
$$

The optimal control exhibits a square root singularity at $t=0$. It is, in fact, Hölder continuous on $[0, T]$ with index $1 / 2$ (see Definition 4.2 below), but not Lipschitz continuous.

So, optimal controls may fail to be Lipschitz continuous when we merely assume (among other relevant hypotheses) that the control constraint map $t \rightarrow U(t)$ is Lipschitz continuous with respect to the Hausdorff metric. On the other hand, the example suggests that we should be seeking rather to establish Hölder continuity-like properties of optimal controls, in these circumstances. This is possible under the following constraint qualification:

(H6A) For the continuous representation of $\bar{u}(\cdot)$ either,

(i) There exists $\varepsilon>0$ such that, for every $t \in[S, T]$ such that $\mathcal{J}(t, \bar{x}(t)) \neq \emptyset$ and for every set of non-negative numbers $\left\{\alpha_{j}\right\}_{j \in \mathcal{J}(t, \bar{x})}$, not all zero, we have

$$
\sum_{j \in \mathcal{J}(t, \bar{x})} \alpha_{j} G^{T}(t, \bar{x}(t)) \nabla_{x} h_{j}(t, \bar{x}(t)) \notin \operatorname{span} \bigcup_{u \in(\bar{u}(t)+\varepsilon B) \cap U(t)} N_{U(t)}(u)
$$

or

(ii) for every $t \in[S, T]$ such that $\mathcal{J}(t, \bar{x}(t)) \neq \emptyset, U(t)$ has a local $C^{1+}$ functional inequality representation (see below) and for every set of non-negative numbers $\left\{\alpha_{j}\right\}_{j \in \mathcal{J}(t, \bar{x})}$, not all zero, we have

$$
\sum_{j \in \mathcal{J}(t, \bar{x})} \alpha_{j} G^{T}(t, \bar{x}(t)) \nabla_{x} h_{j}(t, \bar{x}(t)) \notin \operatorname{span} N_{U(t)}(\bar{u}(t)) .
$$

In the above hypothesis ' $u(t)$ has a local $C^{1+}$ functional inequality representation' means that there exists $\varepsilon>0$ (independent of $t$ ) and $C^{1+}$ functions $\psi_{t}^{i}(\cdot), i=1, \ldots, k$ such that, for each $t \in[S, T]$,

$$
U(t) \cap(\bar{u}(t)+\varepsilon \mathbb{B})=\left\{u \mid \psi_{t}^{i}(u) \leq 0, i=1, \ldots, k\right\} \cap(\bar{u}(t)+\varepsilon \mathbb{B})
$$

and the vectors $\left\{\nabla_{u} \psi_{t}^{i}(t, \bar{u}(t)), i=1, \ldots, k\right\}$ are linearly independent. Note that, in this hypothesis, the functions $t \rightarrow \psi_{t}^{i}(u)$ are not required to be Lipschitz continuous.

Comparing the alternative hypotheses (H6A)(i) and (H6A)(ii), we see that (H6A)(ii) involves a sharper constraint qualification (4.4), because it omits the union operation in (4.3). On the other hand, (H6A)(ii) imposes some additional structure on $U(t)$. 
Definition 4.2 Take a function $v:[S, T] \rightarrow R^{m}$ and $\alpha \in(0,1]$.

(i) $v$ is Hölder continuous with index $\alpha$, if there exists $k \geq 0$ such that

$$
|v(t)-v(s)| \leq k|t-s|^{\alpha} \quad \text { for all }[s, t] \subset[S, T] .
$$

(ii) $v$ is lower Hölder continuous with index $\alpha$, if there exists $k \geq 0$ such that

$$
\liminf _{s_{i}, t_{i} \rightarrow t}\left|t_{i}-s_{i}\right|^{-\alpha}\left|\bar{u}\left(t_{i}\right)-\bar{u}\left(s_{i}\right)\right| \leq k \quad \text { for all } t \in[S, T] .
$$

(In the condition above, the liminf is taken over all sequences $t_{i} \rightarrow t$ and $s_{i} \rightarrow t$ such that $s_{i}<t_{i}$ for each i.)

Lower Hölder continuity is a weaker property than Hölder continuity (for a given index). Note however that, for any $\varepsilon \in(0, \alpha)$, the class of lower Hölder continuous functions (with index $\alpha$ ) excludes functions with an isolated power $\alpha-\varepsilon$ singularity such as

$$
v(t)=|t-S|^{\alpha-\varepsilon} \quad S \leq t \leq T .
$$

Theorem 4.3 Let $(\bar{x}, \bar{u})$ be a normal extremal to $(\mathcal{P})$.

(A) Assume (H1)-(H4) and (H6). Then,

(i) if $r=1, \bar{u}$ is Hölder continuous with index $1 / 2$,

(ii) if $r>1, \bar{u}$ is lower Hölder continuous with index $1 / 2$.

(B) Assume (H1)-(H4) and (H6)(i). Assume further that $U(t)$ is independent of $t$. Then $\bar{u}$ is Lipschitz continuous on $[S, T]$.

(C) Assume (H1)-(H4).Assume further that $r=0$ (no state constraints). Then

(i) $\bar{u}$ is Hölder continuous with index $1 / 2$,

(ii) if $U(t)$ is independent of $t$, then $\bar{u}$ is Lipschitz continuous.

In the preceding theorem, interest focuses on assertion (A). Under the stated conditions involving a time-varying constraint set, optimal controls are Hölder continuous with index $1 / 2$ in the case $r \leq 1$. If however $r>1, \bar{u}$ is known merely to have a weaker regularity property (lower Hölder continuity). The theorem also supplies the information that, in the case $U(t)$ does not depend on time, the above regularity properties can be strengthened to Lipschitz continuity. (This is (B)). Finally, in (C), it asserts that the preceding assertions are valid under weaker hypotheses, when $r=0$ (no state constraints).

Note that Theorem 4.3 (part (C)) predicts that the optimal control for Example 4.1 is Hölder continuous with index $1 / 2$, as observed.

\section{$5 \quad$ Preliminary Analysis}

Define the extended-real-valued function $L_{0}:[S, T] \times \mathbb{R}^{n} \times \mathbb{R}^{m} \rightarrow \mathbb{R} \cup\{+\infty\}$

$$
L_{0}(t, x, u)=L(t, x, u)+\Psi_{U(t)}(u)
$$


in which $\Psi_{U}$ is the indicator function of the set $U$. Note that, since

$$
\max _{u \in U(t)} \mathcal{H}\left(t, \bar{x}(t), q\left(t^{-}\right), 1\right)=\left\langle q\left(t^{-}\right), f(t, \bar{x}(t))\right\rangle+\max _{u \in R^{m}}\left\{\left\langle q\left(t^{-}\right), G(t, \bar{x}(t)) u\right\rangle-L_{0}(t, \bar{x}(t), u)\right\},
$$

we have from the 'Maximization of the Hamiltonian' condition (2.5) that

$$
\left\langle G^{T}(t, \bar{x}(t)) q\left(t^{-}\right), \bar{u}(t)\right\rangle-L(t, \bar{x}(t), \bar{u}(t))=\max _{u \in \boldsymbol{R}^{m}}\left\{\left\langle G^{T}(t, \bar{x}(t)) q\left(t^{-}\right), u\right\rangle-L_{0}(t, \bar{x}(t), u)\right\} \quad \text { a.e. } t .
$$

By the rules governing subdifferentials of convex functions, this last condition implies that

$$
\bar{u}(t)=\partial_{y} L_{0}^{*}\left(t, \bar{x}(t), G^{T}(t, \bar{x}(t)) q\left(t^{-}\right)\right) \quad \text { a.e. } t \in[S, T] .
$$

Here, $L_{0}^{*}(t, x, \cdot): \mathbb{R}^{m} \rightarrow \mathbb{R} \cup\{+\infty\}$ is the Fenchel dual function of $L_{0}(t, x,$.$) for each (t, x)$ :

$$
L_{0}^{*}(t, x, y):=\max _{u \in R^{m}}\left\{\langle y, u\rangle-L_{0}(t, x, u)\right\} .
$$

The representation (5.2) of the optimal control in terms of the Fenchel dual function $L_{0}^{*}$ has a crucial role in our analysis.

Some important regularity properties of $\partial_{y} L_{0}^{*}$ are gathered together in the following lemma. (The calculations involved in verification of property (iii) are similar to those used in proof of [2], Proposition 1.2.)

\section{Lemma 5.1}

Assume (H1)-(H4). Then

(i) For each $(t, x, y) \in \Omega \times \mathbb{R}^{m}, \partial_{y} L_{0}^{*}(t, x, y)$ is single-valued and continuous. (Write it henceforth $\left.\nabla_{y} L_{0}^{*}(t, x, y)\right)$.

(ii) The function $(t, x, y) \rightarrow \nabla_{y} L_{0}^{*}(t, x, y)$ is Hölder continuous with index $1 / 2$ in $t$ and Lipschitz continuous in $(x, y)$ on bounded subsets of $\Omega \times \mathbb{R}^{m}$ in the following sense: given any $N>0$, there exists a constant $K$ such that for any $(t, x, y),\left(t^{\prime}, x^{\prime}, y^{\prime}\right) \in \Omega \times N \mathbb{B}$

$$
\left|\nabla_{y} L_{0}^{*}\left(t^{\prime}, x^{\prime}, y^{\prime}\right)-\nabla_{y} L_{0}^{*}(t, x, y)\right| \leq K\left(\left|t^{\prime}-t\right|^{1 / 2}+\left|\left(x^{\prime}, y^{\prime}\right)-(x, y)\right|\right) .
$$

(iii) If, additionally, $U(t)$ is independent of $t$ then, for every $N>0, \nabla_{y} L_{0}^{*}(\cdot, \cdot, \cdot)$ is Lipschitz continuous on $\Omega \times N \mathbb{B}$.

\section{Proof.}

Take any $(t, x) \in \Omega$ and $y \in \mathbb{R}^{m}$. The non-emptiness of $\partial_{y} L_{0}^{*}(t, x, y)$ follows from the representation of the subdifferential

$$
\partial_{y} L_{0}^{*}(t, x, y)=\left\{u \mid\langle u, y\rangle-L_{0}(t, x, u)=\max _{v \in \boldsymbol{R}^{m}}\left\{\langle v, y\rangle-L_{0}(t, x, v)\right\}\right\}
$$

and the coercivity of $L$ (see Lemma 3.2 (iii)), which ensures existence of a maximizing $v$ via the fact that the function $v \rightarrow\langle v, y\rangle-L_{0}(t, x, v)$ tends to $-\infty$ as $|v| \rightarrow \infty$. Take any number $N>0$. From boundedness of the set of maximizers in (5.3) we conclude that there exists $K_{1}>0$ such that

$$
\text { " } u^{\prime} \in \partial_{y} L_{0}^{*}\left(t^{\prime}, x^{\prime}, y^{\prime}\right) \quad \text { and } \quad\left(t^{\prime}, x^{\prime}\right) \in \Omega, y^{\prime} \in y+N \mathbb{B} " \quad \Rightarrow \quad \text { " }\left|u^{\prime}\right| \leq K_{1} " .
$$

Take arbitrary $\left(t^{\prime}, x^{\prime}\right) \in \Omega$ and $y^{\prime} \in y+N \mathbb{B}$. Choose also

$$
u \in \partial_{y} L_{0}^{*}(t, x, y) \quad \text { and } \quad u^{\prime} \in \partial_{y} L_{0}^{*}\left(t^{\prime}, x^{\prime}, y^{\prime}\right) .
$$


By a fundamental property of 'convex' subdifferentials (see [10])

$$
y \in \partial_{u} L_{0}(t, x, u) \quad \text { and } \quad y^{\prime} \in \partial_{u} L_{0}\left(t^{\prime}, x^{\prime}, u^{\prime}\right) .
$$

But, since $L(t, x, \cdot)$ is continuously differentiable,

$$
\partial_{u} L_{0}(t, x, u)=\nabla_{u} L(t, x, u)+N_{U(t)}(u) .
$$

It follows that

$$
y=\nabla_{u} L(t, x, u)+e, \quad y^{\prime}=\nabla_{u} L\left(t^{\prime}, x^{\prime}, u^{\prime}\right)+e^{\prime},
$$

for some $e \in N_{U(t)}(u)$ and $e^{\prime} \in N_{U\left(t^{\prime}\right)}\left(u^{\prime}\right)$. Also, by boundedness of $x, x^{\prime}, y, y^{\prime}, u, u^{\prime}$

$$
|e| \leq K_{2},\left|e^{\prime}\right| \leq K_{2}
$$

for some $K_{2}$ independent of the choice of $(t, x, u, y)$ and and $\left(t^{\prime}, x^{\prime}, u^{\prime}, y^{\prime}\right)$.

Choose $\tilde{u} \in U(t)$ and $\tilde{u}^{\prime} \in U\left(t^{\prime}\right)$ such that

$$
\max \left\{\left|\tilde{u}-u^{\prime}\right|,\left|\tilde{u}^{\prime}-u\right|\right\} \leq K_{3}\left|t^{\prime}-t\right|
$$

for some $K_{3}$ independent of the choice of $t, t^{\prime}$. Such choice is possible in view of the Lipschitz continuity of $U(t)$ with respect to the Hausdorff metric. We have

$$
\begin{aligned}
& \left\langle y^{\prime}-y, u^{\prime}-u\right\rangle=\left\langle\nabla_{u} L\left(t^{\prime}, x^{\prime}, u^{\prime}\right)-\nabla_{u} L(t, x, u)+e^{\prime}-e, u^{\prime}-u\right\rangle \\
& =\left\langle\nabla_{u} L\left(t, x, u^{\prime}\right)-\nabla_{u} L(t, x, u), u^{\prime}-u\right\rangle+\left\langle\nabla_{u} L\left(t^{\prime}, x^{\prime}, u^{\prime}\right)-\nabla_{u} L\left(t, x, u^{\prime}\right), u^{\prime}-u\right\rangle \\
& -\langle e, \tilde{u}-u\rangle-\left\langle e^{\prime}, \tilde{u}^{\prime}-u^{\prime}\right\rangle+\left\langle e, \tilde{u}-u^{\prime}\right\rangle+\left\langle e^{\prime}, \tilde{u}^{\prime}-u\right\rangle .
\end{aligned}
$$

By (3.2),

$$
\left\langle\nabla_{u} L\left(t, x, u^{\prime}\right)-\nabla_{u} L(t, x, u), u^{\prime}-u\right\rangle \geq \sigma\left|u^{\prime}-u\right|^{2}
$$

( $\sigma$ is the constant of $(\mathrm{H} 4)$ ). Since $\nabla_{u} L$ is locally Lipschitz continuous with respect to the $(t, x)$ variables (see (H4)), there exist $K_{4}$, independent of our choice of $(t, x, y, u)$ and $\left(t^{\prime}, x^{\prime}, y^{\prime}, u^{\prime}\right)$, such that

$$
\left\langle\nabla_{u} L\left(t^{\prime}, x^{\prime}, u^{\prime}\right)-\nabla_{u} L\left(t, x, u^{\prime}\right), u^{\prime}-u\right\rangle \geq-K_{4}\left|\left(t^{\prime}, x^{\prime}\right)-(t, x)\right|\left|u^{\prime}-u\right| .
$$

Also,

$$
\max \left\{\left|\left\langle e, \tilde{u}-u^{\prime}\right\rangle\right|,\left|\left\langle e^{\prime}, \tilde{u}^{\prime}-u\right\rangle\right|\right\} \leq K_{2} K_{3}\left|t^{\prime}-t\right|
$$

and, by the definition of the normal cone of convex analysis

$$
\langle e, \tilde{u}-u\rangle \geq 0, \quad\left\langle e^{\prime}, \tilde{u}^{\prime}-u^{\prime}\right\rangle \geq 0 .
$$

We deduce from (5.4) that

$$
\left|y^{\prime}-y\right|\left|u^{\prime}-u\right| \geq\left\langle y^{\prime}-y, u^{\prime}-u\right\rangle \geq \sigma\left|u^{\prime}-u\right|^{2}-K_{4}\left|\left(t^{\prime}, x^{\prime}\right)-(t, x)\right|\left|u^{\prime}-u\right|-2 K_{1} K_{3}\left|t^{\prime}-t\right| .
$$

or, equivalently,

$$
\sigma\left|u^{\prime}-u\right|^{2}-\left(\left|y^{\prime}-y\right|+K_{4}\left|\left(t^{\prime}, x^{\prime}\right)-(t, x)\right|\right)\left|u^{\prime}-u\right|-2 K_{1} K_{3}\left|t^{\prime}-t\right| \leq 0 .
$$

Completing the square and applying standard estimates yields

$$
\left|u^{\prime}-u\right| \leq \sigma^{-1}\left[\left|y^{\prime}-y\right|+K_{4}\left(\left|x^{\prime}-x\right|+\left|t^{\prime}-t\right|+\left(2 \rho^{-1} K_{4} K_{2}\right)^{1 / 2}\left|t^{\prime}-t\right|^{1 / 2}\right)\right] .
$$


This implies the existence of $K_{5}$ independent of the choice of $(t, x, y, u)$ and $\left(t^{\prime}, x^{\prime}, y^{\prime}, u^{\prime}\right)$ such that

$$
\left|u^{\prime}-u\right| \leq K_{5}\left(\left|\left(x^{\prime}, y^{\prime}\right)-(x, y)\right|+\left|t^{\prime}-t\right|^{1 / 2}\right) .
$$

The preceding inequality also implies that $\partial_{y} L_{0}^{*}(t, x, y)$ is single-valued. Since a convex function with a single-valued subdifferential is continuously differentiable, $\nabla_{y} L_{0}^{*}(t, x, \cdot)$ is continuous.

Let us establish assertion (iii) of the lemma. Assume that $U(t)$ is independent of $t$. Now (5.5) is valid with $K_{3}=0$. (This is because $u^{\prime}$ and $u$ coincide with their projections $\tilde{u}$ and $\tilde{u}^{\prime}$.) But then

$$
\left|u^{\prime}-u\right| \leq \sigma^{-1}\left(\left|y^{\prime}-y\right|+K_{4}\left|\left(t^{\prime}, x^{\prime}\right)-(t, x)\right|\right) .
$$

It follows that $\nabla L_{0}^{*}(\cdot, \cdot, \cdot)$ is Lipschitz continuous on $\Omega \times N \mathbb{B}$.

As a direct consequence of Lemma 5.1, the representation of $\bar{u}$ given by (5.2), the fact that $q(\cdot)$ is a function of bounded variation and the Maximum Principle conditions we deduce (c.f. [2]):

Lemma 5.2 Assume (H1)-(H4). Then

(a) we can choose $\bar{u}$ (from the equivalence class of a.e. equal functions) to have left and right limits at all points in $(S, T)$, one sided limits at the endpoints and (this representation of) $\bar{u}$ is a bounded function, and

(b) the functions $\bar{x}$ and $p$ are Lipschitz continuous.

In the next lemma we establish, moreover, that $\bar{u}$ is continuous and that the measures $\mu_{j}, j=1, \ldots, r$ have no atoms.

\section{Lemma 5.3}

(i) Assume (H1)-(H4). Then $\bar{u}$ is continuous.

(ii) Assume also either (H6A) or (H5) and (H6). Then the $\mu_{j}$ 's have no atoms on $(S, T)$. Furthermore, we have, for each $t \in(S, T)$ and $j \in \mathcal{J}(t, \bar{x}(t))$,

$$
\nabla_{t} h_{j}(t, \bar{x}(t))+\left\langle\nabla_{x} h_{j}(t, \bar{x}(t)), f(t, \bar{x}(t))+G(t, \bar{x}) \bar{u}(t)\right\rangle=0 .
$$

Proof. Take any $t \in(S, T)$. To simplify notation, we shall write $\nabla_{y} L_{0}^{*}(t, x, y)$ as $\nabla_{y} L_{0}^{*}(y)$, and suppress the argument $(t, x)$ in expressions involving $G(t, x)$, etc. Let

$$
\begin{aligned}
& y=G^{T}\left(p(t)+\sum_{j=1}^{r} \int_{[S, t]} \nabla_{x} h_{j}(s) \mu_{j}(d s)\right), \\
& \alpha_{j}=\mu_{j}(\{t\}) \text { for } j=1, \ldots, r \text { and } v=G^{T} \sum_{j=1}^{r} \alpha_{j} \nabla_{x} h_{j} .
\end{aligned}
$$

From (5.2) it follows that

$$
\bar{u}\left(t^{+}\right)=\nabla_{y} L_{0}^{*}(y), \quad \bar{u}\left(t^{-}\right)=\nabla_{y} L_{0}^{*}(y-v) .
$$

By a fundamental property of 'convex' subdifferentials

$$
y \in \partial_{u} L_{0}\left(\bar{u}\left(t^{+}\right)\right) \quad \text { and } \quad y-v \in \partial_{u} L_{0}\left(\bar{u}\left(t^{-}\right)\right) .
$$


But, since $L(t, x, \cdot)$ is continuously differentiable,

$$
y=\nabla_{u} L\left(\bar{u}\left(t^{+}\right)\right)+e, \quad y-v=\nabla_{u} L\left(\bar{u}\left(t^{-}\right)\right)+e^{\prime},
$$

for some $e \in N_{U(t)}\left(\bar{u}\left(t^{+}\right)\right)$and $e^{\prime} \in N_{U(t)}\left(\bar{u}\left(t^{-}\right)\right)$. In view of (3.2) there exists $\sigma>0$ such that

$$
\left\langle\nabla_{u} L\left(\bar{u}\left(t^{+}\right)\right)-\nabla_{u} L\left(\bar{u}\left(t^{-}\right)\right), \bar{u}\left(t^{+}\right)-\bar{u}\left(t^{-}\right)\right\rangle \geq \sigma\left|\bar{u}\left(t^{+}\right)-\bar{u}\left(t^{-}\right)\right|^{2}
$$

From (5.7) and properties of normal cones, it follows that

$$
\begin{aligned}
& \left\langle v, \bar{u}\left(t^{+}\right)-\bar{u}\left(t^{-}\right)\right\rangle=\left\langle\nabla_{u} L\left(\bar{u}\left(t^{+}\right)\right)-\nabla_{u} L\left(\bar{u}\left(t^{-}\right)\right), \bar{u}\left(t^{+}\right)-\bar{u}\left(t^{-}\right)\right\rangle+\left\langle e, \bar{u}\left(t^{+}\right)-\bar{u}\left(t^{-}\right)\right\rangle \\
& -\left\langle e^{\prime}, \bar{u}\left(t^{+}\right)-\bar{u}\left(t^{-}\right)\right\rangle \geq\left\langle\nabla_{u} L\left(\bar{u}\left(t^{+}\right)\right)-\nabla_{u} L\left(\bar{u}\left(t^{-}\right)\right), \bar{u}\left(t^{+}\right)-\bar{u}\left(t^{-}\right)\right\rangle \geq \sigma\left|\bar{u}\left(t^{+}\right)-\bar{u}\left(t^{-}\right)\right|^{2} .
\end{aligned}
$$

Take any $j \in \mathcal{J}(t, \bar{x})$, then $h_{j}(t, \bar{x}(t))=0$. It follows

$$
\delta^{-1}\left(h_{j}(t+\delta, \bar{x}(t+\delta))-h_{j}(t, \bar{x}(t))\right) \leq 0
$$

and

$$
\delta^{-1}\left(h_{j}(t, \bar{x}(t))-h_{j}(t-\delta, \bar{x}(t-\delta))\right) \geq 0,
$$

for $\delta$ sufficiently small. Passing to the limit as $\delta \downarrow 0$ and recalling that $\bar{u}$ has left and right limits, we obtain

$$
\nabla_{t} h_{j}+\left\langle\nabla_{x} h_{j}, f+G \bar{u}\left(t^{+}\right)\right\rangle \leq 0
$$

and

$$
\nabla_{t} h_{j}+\left\langle\nabla_{x} h_{j}, f+G \bar{u}\left(t^{-}\right)\right\rangle \geq 0 .
$$

(Here, $h_{j}, f$, etc. are evaluated at $(t, \bar{x}(t))$.) Hence

$$
\left\langle\nabla_{x} h_{j}, G\left(\bar{u}\left(t^{+}\right)-\bar{u}\left(t^{-}\right)\right)\right\rangle \leq 0 .
$$

Appropriately weighting and summing this inequality over all $j$ 's in $\mathcal{J}(t, \bar{x})$ gives

$$
\left\langle\sum_{j \in \mathcal{J}(t, \bar{x})} \mu_{j}(\{t\}) G^{T} \nabla_{x} h_{j}, \bar{u}\left(t^{+}\right)-\bar{u}\left(t^{-}\right)\right\rangle \leq 0
$$

or

$$
\left\langle v, \bar{u}\left(t^{+}\right)-\bar{u}\left(t^{-}\right)\right\rangle \leq 0 .
$$

But then from (5.8) it follows that $\bar{u}\left(t^{+}\right)=\bar{u}\left(t^{-}\right)$, i.e. $\bar{u}$ is continuous at $t$. Replacing the values of $\bar{u}$ at $S, T$ by limits from the interior of $[S, T]$, if required, we can arrange that $\bar{u}$ is continuous at all points in $[S, T]$. Notice also that, since $\bar{u}\left(t^{+}\right)=\bar{u}\left(t^{-}\right)$, we have

$$
v=e-e^{\prime} \text { and } e, e^{\prime} \in N_{U(t)}(\bar{u}(t)),
$$

which means that $G^{T} \sum_{j=1}^{r} \alpha_{j} \nabla_{x} h_{j}(t, \bar{x}(t)) \in \operatorname{span} N_{U(t)}(\bar{u}(t))$. If we suppose hypothesis (H6) or (H6A) then $\alpha_{j}=0$ for all $j$. But $\alpha_{j}=\mu_{j}(\{t\}), j=1, \ldots, r$, which implies that $\mu_{j}$ 's have no atoms on $(S, T)$.

Finally, we deduce from we deduce (5.9) and (5.10) that

$$
\nabla_{t} h_{j}(t, \bar{x}(t))+\left\langle\nabla_{x} h_{j}(t, \bar{x}(t)), f(t, \bar{x}(t))+G(t, \bar{x}(t)) \bar{u}(t)\right\rangle=0 .
$$

In view of the preceding lemma, we can unambiguously write $\int_{[s, t]} \mu_{j}(d \sigma)$ as $\int_{s}^{t} \mu_{j}(d \sigma)$, for any $[s, t] \subset[S, T]$.

It is convenient now to state for future use a 'robust' form of the constraint qualification (H6), which follows from (H6) and (H1) - (H5) via standard compactness arguments. 
Lemma 5.4 Assume (H1)-(H6). Then there exist $\gamma>0$ and $\varepsilon^{\prime}>0$ with the following properties: for every $t \in[S, T] ; y \in R^{n}, v \in R^{m}$, collection of non-negative numbers $\left\{\alpha_{j}\right\}_{j \in \mathcal{J}(t, \bar{x})}$ and numbers $\left\{\beta_{j}\right\}_{j \in \mathcal{T}(t, \bar{u})}$, such that

$$
\begin{aligned}
& |y-\bar{x}(t)|<\varepsilon^{\prime}, \quad|v-\bar{u}(t)|<\varepsilon^{\prime} \\
& \alpha_{j}=0 \text { if } h_{j}(t, \bar{x}(t))<-\varepsilon^{\prime} \\
& \beta_{j}=0 \text { if } d_{j}(t, \bar{u}(t))<-\varepsilon^{\prime}
\end{aligned}
$$

we have

$$
\left|\sum_{j=1}^{r} \alpha_{j} G^{T}(t, y) \nabla_{x} h_{j}(t, y)+\sum_{j=1}^{l} \beta_{j} \nabla_{u} d_{j}(t, v)\right| \geq \gamma\left(\sum_{j=1}^{r} \alpha_{j}+\sum_{j=1}^{l}\left|\beta_{j}\right|\right) .
$$

We require also:

Lemma 5.5 Assume (H1)-(H6) or (H1)-(H4),(H6A). Fix any $K>0$. Then there exist $\bar{\varepsilon}>0$, $\bar{k}>0$ with the following property:

For any $t \in[S, T], y \in \partial_{u} L_{0}(t, \bar{x}(t), \bar{u}(t))$ and any collection of positive numbers $\left\{\alpha_{j}\right\}$, such that

$$
\alpha_{j}=0 \text { if } h_{j}(t, \bar{x}(t))<0
$$

and

$$
\sum_{j=1}^{r} \alpha_{j}<\bar{\varepsilon}, \quad|y| \leq K
$$

we have

$$
\left\langle v, \nabla_{y} L_{0}^{*}(t, \bar{x}(t), y)-\nabla_{y} L_{0}^{*}(t, \bar{x}(t), y-v)\right\rangle \geq \bar{k}\left|\sum_{j=1}^{r} \alpha_{j}\right|^{2},
$$

where $v=G^{T}(t, \bar{x}(t)) \sum_{j} \alpha_{j} \nabla_{x} h_{j}(t, \bar{x}(t))$.

Proof. Assume (H1)-(H6). Take any non-negative numbers $\alpha_{j}, j=1, \ldots, r$, such that

$$
\alpha_{j}=0 \text { if } h_{j}(t, \bar{x}(t))<0
$$

and $\sum_{j} \alpha_{j}<\bar{\varepsilon}(\bar{\varepsilon}$ will be specified shortly). Define $v$ as in the lemma statement.

From the hypotheses of the lemma $\bar{u}(t)=\nabla_{y} L_{0}^{*}(y)$. Set

$$
\bar{u}:=\bar{u}(t)=\nabla_{y} L_{0}^{*}(y), u^{\prime}:=\nabla_{y} L_{0}^{*}(y-v) .
$$

In view of the Lipschitz continuity of $L_{0}^{*}$ with respect to $y$ variable proved in Lemma 5.1 and the assumption on boundedness of $y$, there exists a constant $k_{2}$ such that $\left|\bar{u}-u^{\prime}\right| \leq k_{2}\left|\sum_{j} \alpha_{j}\right|$. Also,

$$
y=\nabla_{u} L(\bar{u})+e, \text { and } y-v=\nabla_{u} L\left(u^{\prime}\right)+e^{\prime} \text { for some } e \in N_{U(t)}(\bar{u}) \text { and } e^{\prime} \in N_{U(t)}\left(u^{\prime}\right) .
$$

Assume that the set $U(t)$ is given by (1.1) and (H5) holds. The representation of the normal cone to a set given by a system of inequality constraints (see, for example [11]) gives

$$
e=\sum_{j} \beta_{j} \nabla_{u} d_{j}(t, \bar{u}) \text { and } e^{\prime}=\sum_{j} \beta_{j}^{\prime} \nabla_{u} d_{j}\left(t, u^{\prime}\right)
$$

where $\beta_{j}, \beta_{j}^{\prime} \geq 0$ and $\beta_{j}, \beta_{j}^{\prime}=0$ if the corresponding constraints are inactive. 
Choose $\bar{\varepsilon}$ in such a way that

$$
\tilde{k} k_{2} \bar{\varepsilon}<\varepsilon^{\prime}
$$

where $\tilde{k}$ is the Lipschitz constant of $d_{j}$ 's and $\varepsilon^{\prime}$ is as in Lemma 5.4.

Assume that $d_{j}(t, \bar{u})<-\varepsilon^{\prime}$. Then

$$
d_{j}\left(t, u^{\prime}\right)=d_{j}(t, \bar{u})+\left(d_{j}\left(t, u^{\prime}\right)-d_{j}(t, \bar{u})\right)<-\varepsilon^{\prime}+\tilde{k} k_{2} \bar{\varepsilon}<0 .
$$

Therefore, for all $j$,

$$
\beta_{j}^{\prime}=0 \text { if } d_{j}(t, \bar{u})<-\varepsilon^{\prime} .
$$

It follows from the special case of Lemma 5.4 in which the $\alpha_{j}$ 's are all zero that

$$
\sum_{j}\left|\beta_{j}^{\prime}\right| \leq \frac{1}{\gamma}\left|e^{\prime}\right| \leq k_{3}
$$

for some constant $k_{3}$ independent of the choice of $t, y, v$.

In view of (3.2),

$$
\left\langle\nabla_{u} L(\bar{u})-\nabla_{u} L\left(u^{\prime}\right), \bar{u}-u^{\prime}\right\rangle \geq \sigma\left|\bar{u}-u^{\prime}\right|^{2}
$$

and, by properties of the normal cone of convex analysis,

$$
\left\langle e^{\prime}, \bar{u}-u^{\prime}\right\rangle \leq 0 \text { and }\left\langle e, u^{\prime}-\bar{u}\right\rangle \leq 0 .
$$

But then, from (5.11),

$$
\left\langle v, \bar{u}-u^{\prime}\right\rangle=\left\langle\nabla_{u} L(\bar{u})-\nabla_{u} L\left(u^{\prime}\right), \bar{u}-u^{\prime}\right\rangle-\left\langle e^{\prime}, \bar{u}-u^{\prime}\right\rangle-\left\langle e, u^{\prime}-\bar{u}\right\rangle \geq \sigma\left|\bar{u}-u^{\prime}\right|^{2} .
$$

Let $P$ be the projection operator onto the orthogonal complement $V^{\perp}$ of the set

$$
V:=\left\{\sum_{j} \tilde{\beta}_{j} \nabla_{u} d_{j}(t, \bar{u}) \mid \tilde{\beta}_{j} \in \mathbb{R}, j=1, \ldots, l \text { and } \tilde{\beta}_{j}=0 \text { if } d_{j}(t, \bar{u})<-\varepsilon^{\prime}\right\} .
$$

From (5.11), the projection of $v$ on $V^{\perp}, \hat{v}$, is

$$
\hat{v}:=P v=P\left[\nabla_{u} L(\bar{u})-\nabla_{u} L\left(u^{\prime}\right)\right]+P e-P e^{\prime} .
$$

Since $P$ is a contraction

$$
\left|P\left[\nabla_{u} L(\bar{u})-\nabla_{u} L\left(u^{\prime}\right)\right]\right| \leq\left|\nabla_{u} L(\bar{u})-\nabla_{u} L\left(u^{\prime}\right)\right| \leq k_{4}\left|\bar{u}-u^{\prime}\right|
$$

with some uniform constant $k_{4}$. Also

$$
P e=0 \text { since } e \in V \text {. }
$$

Note also that

$$
e^{\prime}=\sum_{j} \beta_{j}^{\prime} \nabla_{u} d_{j}(t, \bar{u})+\sum_{j} \beta_{j}^{\prime}\left(\nabla_{u} d_{j}\left(t, u^{\prime}\right)-\nabla_{u} d_{j}(t, \bar{u})\right) .
$$

In view of (5.13) and (5.16)

$$
\sum_{j} \beta_{j}^{\prime} \nabla_{u} d_{j}(t, \bar{u}) \in V
$$

By (5.14) and the local Lipschitz continuity of $\nabla_{u} d_{j}$ s, there exists a uniform constant $k_{5}$ such that

$$
\left|\sum_{j} \beta_{j}^{\prime} P\left(\nabla_{u} d_{j}\left(t, u^{\prime}\right)-\nabla_{u} d_{j}(t, \bar{u})\right)\right| \leq k_{5}\left|\bar{u}-u^{\prime}\right|
$$


It follows from (5.17)-(5.21) that

$$
|\hat{v}| \leq k_{4}\left|\bar{u}-u^{\prime}\right|+0+k_{5}\left|\bar{u}-u^{\prime}\right|=\left(k_{4}+k_{5}\right)\left|\bar{u}-u^{\prime}\right| .
$$

But then, from (5.15),

$$
\left\langle v, \bar{u}-u^{\prime}\right\rangle \geq \frac{\sigma}{\left(k_{4}+k_{5}\right)^{2}}|\hat{v}|^{2} .
$$

By construction, $|\hat{v}|$ is equal to the distance of $v$ from $V$ :

$|\hat{v}|=\operatorname{dist}(v, V)=\min _{w \in V}|v-w|=\min \left\{\left|v-\sum_{j=1}^{l} \tilde{\beta}_{j} \nabla_{u} d_{j}(t, \bar{u})\right|, \tilde{\beta}_{j} \in \mathbb{R}\right.$ and $\tilde{\beta}_{j}=0$ if $\left.d_{j}(t, \bar{u})<-\varepsilon^{\prime}\right\}$.

In view of Lemma 5.4,

$$
|\hat{v}| \geq \gamma\left|\sum_{j} \alpha_{j}\right|
$$

Therefore

$$
\left\langle v, \bar{u}-u^{\prime}\right\rangle \geq \bar{k}\left|\sum_{j} \alpha_{j}\right|^{2}
$$

where $\bar{k}=\frac{\sigma \gamma^{2}}{\left(k_{4}+k_{5}\right)^{2}}$. This is the desired inequality.

Now assume (H1)-(H4) and (H6A). $U(t)$ no longer has representation (1.1), but inequality (5.15) still holds true. In view of the Lipschitz continuity of $\nabla_{u} L$, we deduce from (5.15) that

$$
\left\langle v, \bar{u}-u^{\prime}\right\rangle \geq \frac{\sigma}{k_{L}}\left|\nabla_{u} L(\bar{u})-\nabla_{u} L\left(u^{\prime}\right)\right|^{2},
$$

where $k_{L}$ is the Lipschitz constant of $\nabla_{u} L$.

From (5.11) (which is also valid), we have

$$
\left|\nabla_{u} L(\bar{u})-\nabla_{u} L\left(u^{\prime}\right)\right|=\left|v-e^{\prime}+e\right| .
$$

It can be deduced from (4.3) that for some uniform constant $k_{6}$

$$
\left|v-e^{\prime}+e\right| \geq k_{6}\left|\sum_{j} \alpha_{j}\right|
$$

It follows that

$$
\left\langle v, \bar{u}-u^{\prime}\right\rangle \geq \bar{k}\left|\sum_{j} \alpha_{j}\right|^{2}
$$

where now $\bar{k}=\frac{\sigma k_{6}}{k_{L}^{2}}$. We have, once again, arrived at the desired inequality. The lemma is proved.

We have established regularity properties of $\nabla_{y} L_{0}^{*}$ under hypotheses (H1)-(H4) (local Hölder continuity with respect to $t$ and local Lipschitz continuity with respect to the remaining variables). These may be strengthened, if $U(t)$ has representation (1.1) and we add (H5) to the hypotheses.

Recall that (the continuous representations of) $\bar{u}(\cdot)$ and $q(\cdot)$ are related according to

$$
\bar{u}(t)=\nabla_{y} L_{0}^{*}(t, \bar{x}(t), \bar{y}(t))
$$

for each $t \in[S, T]$, where

$$
\bar{y}(t):=G^{T}(t, \bar{x}(t)) q(t)
$$


Lemma 5.6 Assume (H1)-(H5). Then there exists $\varepsilon^{\prime \prime}>0$ such that $\nabla_{y} L_{0}^{*}$ is Lipschitz continuous on

$$
\left\{(t, x, y) \in[0, T] \times \mathbb{R}^{n} \times \mathbb{R}^{m}||(x, y)-(\bar{x}(t), \bar{y}(t)) \mid \leq \varepsilon^{\prime \prime}\right\}
$$

Proof. We know from representation (5.3) that, for each $(t, x, y) \in \Omega \times \mathbb{R}^{m}, \nabla_{y} L_{0}^{*}(t, x, y)$ is the unique minimizer for the convex optimization problem

$$
P(t, x, y)\left\{\begin{array}{l}
\text { Minimize } L(t, x, u)-\langle y, u\rangle \\
\text { over } u \in \mathbb{R}^{m} \text { satisfying } \\
d_{j}(t, u) \leq 0, \quad j=1, \ldots, l
\end{array}\right.
$$

in which $\alpha=(t, x, y)$ is regarded as a parameter. In view of hypothesis (H4), we may deduce from known Lipschitz stability results (see [9], Thm. 2.4 and 4.1, or [2], Prop. 1.4) that, for each $t \in[S, T]$, there is a neighborhood of $(t, \bar{x}(t), \bar{y}(t))$ on which $\nabla_{y} L_{0}^{*}$ is Lipschitz continuous. (Notice that, in the case $t$ is an end point of the interval $[S, T]$, it is necessary to apply [9] to some suitable extension of $\nabla_{y} L_{0}^{*}$, obtained by constant extrapolation with respect to the $t$ variable.) The assertions of the lemma follow by means of a standard compactness argument.

The final lemma in this section provides bounds on $\sum_{j} \int_{s}^{t} \mu_{j}(d \sigma)$ for any subinterval $[s, t]$ having the property that all constraints that are active at some interior point of the subinterval are active also at both endpoints.

Lemma 5.7 There exist constants $K$ and $K^{\prime}$ with the following properties: let $[s, t] \subset[S, T]$ be such that

$$
h_{j}(s, \bar{x}(s))=h_{j}(t, \bar{x}(t))=0
$$

for all $j$ 's satisfying

$$
h_{j}(\sigma, \bar{x}(\sigma))=0
$$

for some $\sigma \in(s, t)$. Then

(i) If either (H1)-(H6) are satisfied, or $U(t)$ is independent of $t$ and (H1)-(H4), (H6A)(i) are satisfied, we have

$$
\sum_{j} \int_{s}^{t} \mu_{j}(d \sigma) \leq K|t-s|
$$

(ii) If (H1)-(H4, (H6A) are satisfied, we have

$$
\sum_{j} \int_{s}^{t} \mu_{j}(d \sigma) \leq K^{\prime}|t-s|^{\frac{1}{2}}
$$

\section{Proof.}

Assume that either (H1)-(H6), or (H1)-(H4),(H6A)(i), are satisfied and $U(t)$ is independent of $t$. Let $[s, t]$ be an interval with the properties described at the beginning of the lemma statement.

Take any $\tilde{\varepsilon}>0$. It is clear that, when verifying assertions (i) and (ii) of the lemma, we need only consider the case when

$$
\sum_{j} \int_{s}^{t} \mu_{j}(d \sigma) \leq \tilde{\varepsilon}
$$


We know that one of the following two cases must occur:

$$
\begin{array}{ll}
\text { (a) : } & \sum_{j} \int_{s}^{\frac{s+t}{2}} \mu_{j}(d \sigma) \geq \frac{1}{2} \sum_{j} \int_{s}^{t} \mu_{j}(d \sigma) \\
\text { (b) : } & \sum_{j} \int_{\frac{s+t}{2}}^{t} \mu_{j}(d \sigma) \geq \frac{1}{2} \sum_{j} \int_{s}^{t} \mu_{j}(d \sigma) .
\end{array}
$$

We assume (a); minor changes to the ensuing analysis, which we omit, can be made to cover the case (b). Define

$$
P=\int_{s}^{t} \sum_{j}\left(h_{j}(t, \bar{x}(t))-h_{j}(\sigma, \bar{x}(\sigma))\right) \mu_{j}(d \sigma) .
$$

Note that, if $j$ is an index value such that $\int_{s}^{t} \mu_{j}(d \sigma) \neq 0$, then $h_{j}(t, \bar{x}(t))=0$. Also, for any $j$,

$$
\operatorname{supp}\left\{\mu_{j}\right\} \subset\left\{\sigma \mid h_{j}(\sigma, \bar{x}(\sigma))=0\right\} .
$$

It follows that

$$
P=0
$$

Writing

$$
h_{j}(t, \bar{x}(t))-h_{j}(\sigma, \bar{x}(\sigma))=\int_{\sigma}^{t}\left(\nabla_{t} h_{j}+\left\langle\nabla_{x} h_{j}, f+G \bar{u}(\tau)\right\rangle\right) d \tau
$$

substituting it into (5.25) and interchanging the order of integration gives

$$
P=\int_{s}^{t} d \tau \int_{s}^{\tau} \sum_{j}\left(\nabla_{t} h_{j}+\left\langle\nabla_{x} h_{j}, f+G \bar{u}(\tau)\right\rangle\right) \mu_{j}(d \sigma) .
$$

(Here, and below, $\nabla_{t} h_{j}, \nabla_{x} h_{j}, f$ and $G$ are evaluated at $\left.(\tau, \bar{x}(\tau))\right)$. But

$$
P=a+b,
$$

where

$$
a=\int_{s}^{t} d \tau \int_{s}^{\tau} \sum_{j}\left(\nabla_{t} h_{j}+\left\langle\nabla_{x} h_{j}, f+G v(\tau)\right\rangle\right) \mu_{j}(d \sigma)
$$

and

$$
b=\int_{s}^{t} d \tau \int_{s}^{\tau} \sum_{j}\left\langle\nabla_{x} h_{j}, G(\bar{u}(\tau)-v(\tau))\right\rangle \mu_{j}(d \sigma) .
$$

In these formulas,

$v(\tau):=\nabla_{y} L_{0}^{*}\left(\tau, \bar{x}(\tau), G^{T}\left[p(\tau)+\int_{S}^{\tau} \sum_{j} \nabla_{x} h_{j}(\sigma, \bar{x}(\sigma)) \mu_{j}(d \sigma)-\sum_{j} \nabla_{x} h_{j}(\tau, \bar{x}(\tau)) \int_{s}^{\tau} \mu_{j}(d \sigma)\right]\right)$.

Our next goal is to show that

$$
b \geq k_{1} \int_{s}^{t}\left|\sum_{j} \int_{s}^{\tau} \mu_{j}(d \sigma)\right|^{2} d \tau .
$$

Denote

$$
\begin{aligned}
y & \left.:=G^{T}\left[p(\tau)+\int_{S}^{\tau} \sum_{j} \nabla_{x} h_{j}(\sigma, \bar{x}(\sigma)) \mu_{j}(d \sigma)\right]\right), \\
w & :=G^{T} \sum_{j} \nabla_{x} h_{j}(\tau, \bar{x}(\tau)) \int_{s}^{\tau} \mu_{j}(d \sigma)
\end{aligned}
$$


Then

$$
\bar{u}(\tau)=\nabla_{y} L_{0}^{*}(\tau, \bar{x}(\tau), y), v(\tau)=\nabla_{y} L_{0}^{*}(\tau, \bar{x}(\tau), y-w)
$$

and

$$
b=\int_{s}^{t}\left\langle G^{T} \sum_{j} \nabla_{x} h_{j} \int_{s}^{\tau} \mu_{j}(d \sigma), \nabla_{y} L_{0}^{*}(y)-\nabla_{y} L_{0}^{*}(y-w)\right\rangle d \tau=\int_{s}^{t}\left\langle w, \nabla_{x} L_{0}^{*}(y)-\nabla_{x} L_{0}^{*}(y-w)\right\rangle d \tau
$$

By choosing $\tilde{\varepsilon}$ in (5.22) appropriately, we can arrange that $\sum_{j} \int_{s}^{t} \mu_{j}(d \sigma)$ is arbitrarily small. It follows then from Lemma 5.5 that there exists $k_{1}$, independent of $[s, t]$ such that $(5.27)$ holds true. But then, by (5.23),

$$
\begin{aligned}
b & \geq k_{1} \int_{\frac{t+s}{2}}^{t}\left|\sum_{j} \int_{s}^{\tau} \mu_{j}(d \sigma)\right|^{2} d \tau \geq k_{1} \int_{\frac{t+s}{2}}^{t}\left|\sum_{j} \int_{s}^{\frac{t+s}{2}} \mu_{j}(d \sigma)\right|^{2} d \tau \\
& \geq\left(k_{1} / 4\right)\left|\sum_{j} \int_{s}^{t} \mu_{j}(d \sigma)\right|^{2} \int_{\frac{t+s}{2}}^{t} d \tau=\left(k_{1} / 8\right)\left|\sum_{j} \int_{s}^{t} \mu_{j}(d \sigma)\right|^{2}|t-s|,
\end{aligned}
$$

Note that, for any index value $j$ such that $\int_{s}^{t} \mu_{j}(d \sigma) \neq 0$ we have $j \in \mathcal{J}(s, \bar{x}(s))$ under our hypotheses. Since $v(s)=\bar{u}(s)$, we have from (5.6)

$$
0=\nabla_{t} h_{j}(s, \bar{x}(s))+\left\langle\nabla_{x} h_{j}(s, \bar{x}(s), f(s, \bar{x}(s))+G(s, \bar{x}(s)) v(s))\right\rangle .
$$

Since $f, G$ and the derivatives of the $h_{j}$ 's are locally Lipschitz continuous, it follows from (5.29) that there exists $k_{2}>0$ (independent of $[s, t]$ ) such that, for all $\tau \in[s, t]$,

$$
\left|\nabla_{t} h_{j}(\tau, \bar{x}(\tau))+\left\langle\nabla_{x} h_{j}(\tau, \bar{x}(\tau), f(\tau, \bar{x}(\tau))+G(\tau, \bar{x}(\tau)) v(\tau))\right\rangle\right| \leq k_{2}(|\tau-s|+|v(\tau)-v(s)|)
$$

We note next that

$$
\begin{aligned}
& |v(\tau)-v(s)| \leq \\
& \left.\mid \nabla_{y} L_{0}^{*}\left(\tau, \bar{x}(\tau), G^{T}\left[p(\tau)+\int_{S}^{\tau} \sum_{j} \nabla_{x} h_{j}(\sigma, \bar{x}(\sigma)) \mu_{j}(d \sigma)\right]-\sum_{j} \nabla_{x} h_{j}(\tau, \bar{x}(\tau)) \int_{s}^{\tau} \mu_{j}(d \sigma)\right]\right) \\
& -\nabla_{y} L_{0}^{*}\left(s, \bar{x}(s), G^{T}\left[p(s)+\int_{S}^{s} \sum_{j} \nabla_{x} h_{j}(\sigma, \bar{x}(\sigma)) \mu_{j}(d \sigma)\right]\right) \mid .
\end{aligned}
$$

At this stage, it is necessary to distinguish two cases:

Case (i): Either (H1)-(H6) are satisfied or (H1)-(H4), (H6A)(i) are satisfied and $U(t)$ is independent of $t$.

In this case, we deduce from (5.31), employing the Lipschitz continuity of $p(\cdot)$ and $\bar{x}(\cdot)$, and either Lemma 5.1 or Lemma 5.6 (depending on which hypotheses are invoked), concerning the Lipschitz continuity properties of $\nabla_{y} L_{0}^{*}(\cdot, \cdot, \cdot)$, that there exists $k_{3}>0$ (independent of $[s, t]$ ) such that, for all $\tau \in[s, t]$,

$$
|v(\tau)-v(s)|<k_{3}\left(1+\sum_{j} \int_{s}^{t} \mu_{j}(d \sigma)\right)(\tau-s) .
$$

From (5.30) then,

$$
\left|\nabla_{t} h_{j}(\tau, \bar{x}(\tau))+\left\langle\nabla_{x} h_{j}(\tau, \bar{x}(\tau), f(\tau, \bar{x}(\tau))+G(\tau, \bar{x}(\tau)) v(\tau))\right\rangle\right| \leq k_{4}\left(1+\sum_{j} \int_{s}^{t} \mu_{j}(d \sigma)\right)(\tau-s)
$$


(for some $k_{4}>0$ independent of $[s, t]$ ). But then

$$
a \geq-\left(k_{4} / 2\right) \sum_{j} \int_{s}^{t} \mu_{j}(d \sigma)\left(1+\sum_{j} \int_{s}^{t} \mu_{j}(d \sigma)\right)(t-s)^{2} .
$$

By (5.26),

$$
\begin{aligned}
0 & =a+b \\
& \geq-\left(k_{4} / 2\right) \sum_{j} \int_{s}^{t} \mu_{j}(d \sigma)\left(1+\sum_{j} \int_{s}^{t} \mu_{j}(d \sigma)\right)(t-s)^{2}+\left(k_{1} / 8\right)\left|\sum_{j} \int_{s}^{t} \mu_{j}(d \sigma)\right|^{2}|t-s| .
\end{aligned}
$$

This implies that

$$
\left|\sum_{j} \int_{s}^{t} \mu_{j}(d \sigma)\right| \leq K|t-s|
$$

where $K=4\left(k_{4} / k_{1}\right)(1+\tilde{\varepsilon})$. This is the desired inequality.

Case (ii): (H1)-(H4) and (H6A) are satisfied.

In this case, we deduce from Lemma 5.1 and (5.31) that, for some $k_{5}>0$ (independent of $[s, t])$ and all $\tau \in[s, t]$

$$
|v(\tau)-v(s)|<k_{5}\left(|\tau-s|^{\frac{1}{2}}+\sum_{j} \int_{s}^{t} \mu_{j}(d \sigma)(\tau-s)\right) .
$$

From (5.30) we have then, for every $\tau \in[s, t]$,

$$
\begin{aligned}
& \left|\nabla_{t} h_{j}(\tau, \bar{x}(\tau))+\left\langle\nabla_{x} h_{j}(\tau, \bar{x}(\tau), f(\tau, \bar{x}(\tau))+G(\tau, \bar{x}(\tau)) u(\tau))\right\rangle\right| \\
& \quad \leq k_{6}\left(|\tau-s|^{\frac{1}{2}}+\sum_{j} \int_{s}^{t} \mu_{j}(d \sigma)(\tau-s)\right)
\end{aligned}
$$

(for some $k_{6}>0$ independent of $[s, t]$ ). It follows that

$$
a \geq-k_{7} \sum_{j} \int_{s}^{t} \mu_{j}(d \sigma)\left(|t-s|^{\frac{3}{2}}+\sum_{j} \int_{s}^{t} \mu_{j}(d \sigma)(t-s)^{2}\right) .
$$

(for some $k_{7}$ independent of $[s, t]$ ). From (5.26) then

$$
\begin{aligned}
0= & a+b \\
& \geq\left(\sum_{j} \int_{s}^{t} \mu_{j}(d \sigma)|t-s|\right) \times \\
& \left(\frac{k_{1}}{8}\left|\sum_{j} \int_{s}^{t} \mu_{j}(d \sigma)\right|-k_{7}\left(|t-s|^{\frac{1}{2}}+\sum_{j} \int_{s}^{t} \mu_{j}(d \sigma)|t-s|\right)\right) .
\end{aligned}
$$

But then

$$
\left|\sum_{j} \int_{s}^{t} \mu_{j}(d \sigma)\right| \leq K^{\prime}|t-s|^{\frac{1}{2}}
$$

where

$$
K^{\prime}=8\left(k_{7} / k_{1}\right)(1+\tilde{\varepsilon}|T-S|) .
$$

We have arrived at the desired inequality in this case also. The proof is complete. 


\section{Proofs of Theorem 3.2 and Theorem 4.3}

Assume first the hypotheses (H1)-(H6) of Theorem 3.2 (or assume that (H1)-(H4), (H6A)(i) are satisfied and $U(t)$ is independent of $t$ as in the last part of Theorem 4.3). We must show that $\bar{u}$ is Lipschitz continuous. The analysis centers on finding a constant $K>0$, such that

$$
\sum_{j} \int_{s}^{t} \mu_{j}(d \sigma) \leq K|t-s| .
$$

for all intervals $[s, t] \subset[S, T]$. Such an estimate is already available to us (see Lemma 5.7) when any state constraint that is active at an interior point is satisfied also at both endpoints. Our immediate task is to remove this restriction.

It is helpful at this stage to introduce some fresh notation. Define

$$
\mathcal{A}_{[s, t]}:=\left\{j \in\{1, \ldots, r\} \mid h_{j}(\tau, \bar{x}(\tau))=0 \text { for some } \tau \in(s, t)\right\} .
$$

('the set of indices corresponding to state constraints that are active at some point in $(s, t)^{\prime}$ ) and

$$
\mathcal{N}_{[s, t]}:=\operatorname{cardinality}\left(\mathcal{A}_{[s, t]}\right) .
$$

For $\bar{r} \in\{0, \ldots, r\}$ denote by $\left(H_{\bar{r}}\right)$ the condition

$\left(H_{\bar{r}}\right)$ : there exists $K_{\bar{r}} \geq 0$ with the property: given any subinterval $[s, t] \subset[S, T]$ such that $\mathcal{N}_{[s, t]} \leq \bar{r}$ we have

$$
\sum_{j} \int_{[s, t]} \mu_{j}(d \sigma) \leq K_{\bar{r}}|t-s|
$$

Lemma 6.1 Assume (H1)-(H6), or assume (H1)-(H4), (H6A)(i) and $U(t)$ is independent of $t$. Then there exists $K>0$ such that

$$
\sum_{j} \int_{[s, t]} \mu_{j}(d \sigma) \leq K|t-s|
$$

for all $[s, t] \subset[S, T]$.

Proof. The assertions of the lemma will follow immediately, if we can show that $\left(H_{\bar{r}}\right)$ is satisfied for for $\bar{r}=r$ (the number of state inequality constraints). We confirm this property by induction. $\left(H_{\bar{r}}\right)$ is true for $\bar{r}=0$ since, in this case, $\mu_{j}=0$ for all $j \in\{1, \ldots, r\}$. Fix $\bar{r} \in\{0, \ldots, r-1\}$ and assume that

$$
\left(H_{\bar{r}}\right) \text { is true . }
$$

We shall show that $\left(H_{\bar{r}+1}\right)$ is true; this will complete the proof.

Take any $[s, t] \subset[S, T]$ such that $\mathcal{N}_{[s, t]} \leq \bar{r}+1$.We must find $K_{\bar{r}+1}$ (independent of $[s, t]$ ) such that

$$
\sum_{j} \int_{[s, t]} \mu_{j}(d \sigma) \leq K_{\bar{r}+1}|t-s| .
$$

We can assume that $\mathcal{N}_{[s, t]}=\bar{r}+1$ for, otherwise, (6.32) is true with $K_{\bar{r}+1}=K_{\bar{r}}$. Our next goal is to find a point $\bar{s} \in[s, t]$ such that

$$
\sum_{j} \int_{[s, \bar{s}]} \mu_{j}(d \sigma) \leq K_{\bar{r}}|\bar{s}-s|
$$

and either of the following two conditions holds: 
(a): $\bar{s}=t$, or

(b): $h_{j}(\bar{s}, \bar{x}(\bar{s}))=0$ for all $j \in \mathcal{A}_{[s, t]}$.

If $h_{j}(s, \bar{x}(s))=0$ for all $j \in \mathcal{A}_{[s, t]}$, we can set $\bar{s}=s$, and (6.33) and condition (b) are satisfied. So we can assume that

$$
h_{j}(\bar{s}, \bar{x}(\bar{s}))<0 \text { for some } j \in \mathcal{A}_{[s, t]} .
$$

We now construct an increasing sequence $\left\{s_{i}\right\} \subset(s, t]$ that terminates after $N$ steps, in which case we set $\bar{s}=s_{N}$, or which is an infinite sequence, in which case we set $\bar{s}=\lim _{i \rightarrow \infty} s_{i}$. In either case, $\bar{s}$ will have the desired properties, as we now confirm.

Define

$$
s_{1}=\sup _{\sigma \in(s, t)}\left\{\sigma: \mathcal{N}_{[s, \sigma]} \leq \bar{r}\right\}
$$

By condition (6.34), $s_{1}>s$. We have

$$
\sum_{j} \int_{\left[s, s_{1}\right]} \mu_{j}(d \sigma) \leq K_{\bar{r}}\left|s_{1}-s\right| .
$$

If $s_{1}=t$, set $\bar{s}=s_{1}$. Then condition (a) is satisfied, and so is (6.33), by the induction hypothesis. If $s_{1}<t$ and

$$
h_{j}\left(s_{1}, \bar{x}\left(s_{1}\right)\right)=0 \quad \text { for all } j \in \mathcal{A}_{[s, t]}
$$

also set $\bar{s}=s_{1}$. In this case condition (b) and (6.33) are satified. Otherwise $s_{1}<t$ and

$$
h_{\bar{j}}\left(s_{1}, \bar{x}\left(s_{1}\right)\right)<0 \quad \text { for some } \bar{j} \in \mathcal{A}_{[s, t]} .
$$

In this case define $s_{2}\left(>s_{1}\right)$ to be

$$
s_{2}=\sup _{\sigma \in\left(s_{1}, t\right)}\left\{\sigma: \mathcal{N}_{\left[s_{1}, \sigma\right]} \leq \bar{r}\right\}
$$

By the induction hypothesis $\sum_{j} \int_{\left[s_{1}, s_{2}\right]} \mu_{j}(d s) \leq K_{\bar{r}}\left|s_{2}-s_{1}\right|$, from which we conclude that

$$
\sum_{j} \int_{\left[s, s_{2}\right]} \mu_{j}(d \sigma) \leq \sum_{j} \int_{\left[s, s_{1}\right]} \mu_{j}(d \sigma)+\sum_{j} \int_{\left[s_{1}, s_{2}\right]} \mu_{j}(d s) \leq K_{\bar{r}}\left|s_{2}-s\right| .
$$

Observe also that, if $s_{2}<t$,

$$
\max _{\sigma \in\left[s_{1}, s_{2}\right]} h_{j}(\sigma, \bar{x}(\sigma))=0 \quad \text { for all } j \in \mathcal{A}_{[s, t]}
$$

for, otherwise, $s_{2}$ cannot provide the supremum in (6.35). If $s_{2}=t$, set $\bar{s}=s_{2}$. In this case (6.33) and condition (a) are satisfied. If $s_{2}<t$ and $h_{j}\left(s_{2}, \bar{x}\left(s_{2}\right)\right)=0$ for all $j \in \mathcal{A}_{[s, t]}$, set $\bar{s}=s_{2}$; (6.33) and condition (b) are satisfied.

If neither condition (a) nor (b) are satisfied (when $\bar{s}=s_{2}$ ), construct $s_{3} \in\left(s_{2}, t\right]$, and so on.

This procedure provides either an element $\bar{s} \in(s, t]$ satisfying (6.33) and either condition (a) or (b) in a fin te number of steps, or it generates an infinite increasing sequence $\left\{s_{i}\right\}$ in $(s, t]$. In the latter case

$$
\sum_{j} \int_{\left[s, s_{i}\right]} \mu_{j}(d \sigma) \leq K_{\bar{r}}\left|s_{i}-s\right| \text { for all } i
$$

and

$$
\max _{s_{i} \leq \sigma \leq s_{i+1}} h_{j}(\sigma, \bar{x}(\sigma))=0 \quad \text { for all } j \in \mathcal{A}_{[s, t]} .
$$


Let $\bar{s}=\lim _{i \rightarrow \infty} s_{i}$. We have $\bar{s} \in(s, t]$. Furthermore, the preceding relationships ensure that

$$
\sum_{j} \int_{[s, \bar{s}]} \mu_{j}(d \sigma) \leq K_{\bar{r}}|\bar{s}-s|
$$

and

$$
h_{j}(\bar{s}, \bar{x}(\bar{s}))=0 \quad \text { for all } j \in \mathcal{A}_{[s, t]} .
$$

Similarly, working from the right endpoint of $[s, t]$, we can find $\bar{t} \in[s, t]$ such that

$$
\sum_{j} \int_{[\bar{t}, t]} \mu_{j}(d \sigma) \leq K_{\bar{r}}|t-\bar{t}|
$$

and either

$\left(\mathrm{a}^{\prime}:\right) \bar{t}=s$, or

$\left(\mathrm{b}^{\prime}\right): h_{j}(\bar{t}, \bar{x}(\bar{t}))=0 \quad$ for all $j \in \mathcal{A}_{[s, t]} \cdot$

If either (a) or $\left(\mathrm{a}^{\prime}\right)$ are true, then (6.32) is true with $K_{\bar{r}+1}=K_{\bar{r}}$. If, on the other hand, $\bar{s}<t$ and $s<\bar{t}$, then $\bar{s} \leq \bar{t}$ and

$$
h_{j}(\bar{s}, \bar{x}(\bar{s}))=h_{j}(\bar{t}, \bar{x}(\bar{t}))=0 \quad \text { for all } j \in \mathcal{A}_{[s, t]} .
$$

It follows from Lemma 5.6 that, for some $K>0$ (that does not depend on $[s, t]$ ),

$$
\sum_{j} \int_{[\bar{s}, \bar{t}]} \mu_{j}(d \sigma) \leq K|\bar{t}-\bar{s}|
$$

But then by (6.36) and (6.37),

$$
\sum_{j} \int_{[s, t]} \mu_{j}(d \sigma)=\sum_{j} \int_{[s, \bar{s}] \cup[\bar{s}, \bar{t}] \cup[\bar{s}, t]} \mu_{j}(d \sigma) \leq \tilde{K}|t-s|,
$$

where $\tilde{K}=\max \left\{K, K_{r}\right\}$. Since $\tilde{K}$ does not depend on $[s, t]$, the lemma is proved.

It is now a simple matter to complete the proof of Thm. 3.2 and Thm. 4.3 (B). By Lemma 6.1 and since $\bar{x}($.$) and p($.$) are Lipschitz continuous, it follows that t \rightarrow\left(t, \bar{x}(t), G^{T}(t, \bar{x}(t)) q(t)\right)$ is Lipschitz continuous on $[S, T]$. In view of Lemmas 5.7, 5.1 and $6.1, \bar{u}$ can be expressed as the composition of two Lipschitz continuous functions, thus

$$
\bar{u}(t)=\nabla_{y} L_{0}^{*} \circ\left(t, \bar{x}(t), G^{T}(t, \bar{x}(t)) q(t)\right),
$$

and is therefore a Lipschitz continuous function. Thm. 3.2 and Thm. 4.3(B) are proved.

We now turn to Thm. 4.3. We have already dealt with assertion (B). Suppose that $r=0$ (no state constraints) and merely (H1)-(H4) are satisfied. Then $q($.$) is Lipschitz continuous.$ So, in view of Lemma 5.1 and the representation (6.38), $\bar{u}$ is Hölder continuous with index 1/2. If additonally $U(t)$ does not depend on time, $\nabla_{y} L_{0}^{*}$ is Lipschitz continuous. It follows that $\bar{u}$ is Lipschitz continuous. (C) is proved.

It remains then to attend to assertion (A). Assume then (H1)-(H4) and (H6). We shall require:

Lemma 6.2 Assume (H1)-(H4) and (H6). 
(i) if $r=1$, there exists $\bar{K} \geq 0$ such that

$$
\sum_{j} \int_{s}^{t} \mu_{j}(d s) \leq \bar{K}|t-s| \quad \text { for all }[s, t] \subset[S, T] .
$$

(ii) if $r>1$, there exists $\bar{K} \geq 0$ such that, given any nontrivial interval $[s, t] \subset[S, T]$, a nontrivial subinterval $\left[s^{\prime}, t^{\prime}\right] \subset[s, t]$ can be found such that

$$
\sum_{j} \int_{s^{\prime}}^{t^{\prime}} \mu_{j}(d s) \leq \bar{K}\left|t^{\prime}-s^{\prime}\right| .
$$

\section{Proof.}

(i) Assume $r=1$. Take any nontrivial interval $[s, t] \subset[S, T]$. If the state constraint is inactive on $(s, t)$ then $\mu_{1}([s, t])=0$ and there is nothing to prove. So suppose that the state constraint is active at some point in $(s, t)$. We can then define

$$
\begin{aligned}
& s^{*}=\inf \left\{\sigma \in[s, t] \mid h_{1}(\sigma, x(\sigma))=0\right\} \\
& t^{*}=\inf \left\{\tau \in[s, t] \mid h_{1}(\tau, x(\tau))=0\right\} .
\end{aligned}
$$

Then $s \leq s^{*} \leq t^{*} \leq t$ and the state constraint is inactive on the intervals $\left[s, s^{*}\right)$ and $\left(t^{*}, t\right]$. Furthermore, $h_{1}\left(s^{*}, \bar{x}\left(s^{*}\right)\right)=h_{1}\left(t^{*}, \bar{x}\left(t^{*}\right)\right)=0$. It follows that

$$
\int_{s}^{t} \mu_{1}(d s)=\int_{s^{*}}^{t^{*}} \mu_{1}(d s) \leq K^{\prime}\left|t^{*}-s^{*}\right|^{1 / 2} \leq K^{\prime}|t-s|^{1 / 2}
$$

where $K^{\prime}$ is the constant of Lemma 5.7.

(ii) We recall that $\mathcal{A}_{[s, t]}$ denotes the set of index values of state constraints that are active at some point in $(s, t)$ and that $\mathcal{N}_{[s, t]}$ is the cardinality of this set. For $\bar{r} \in\{0,1, \ldots, r\}$ let $\left(\tilde{H}_{\bar{r}}\right)$ be the condition:

$\left(\tilde{H}_{\bar{r}}\right)$ : there exists $K_{\bar{r}}>0$ with the property that, given any non-trivial interval $[s, t] \subset[S, T]$ such that $\mathcal{N}_{[s, t]} \leq r$, a non-trivial subinterval $\left[s^{\prime}, t^{\prime}\right] \subset[s, t]$ can be found such that

$$
\sum_{j} \int_{s^{\prime}}^{t^{\prime}} \mu_{j}(d \sigma) \leq K_{\bar{r}}\left|t^{\prime}-s^{\prime}\right|^{1 / 2}
$$

We must show that $\tilde{H}_{\bar{r}}$ is true for $\bar{r}=r$. But this is proved by induction. $\left(\tilde{H}_{\bar{r}}\right)$ is trivially true for $\bar{r}=0$. Suppose that, for some $\bar{r} \in\{0,1, \ldots, r-1\},\left(\tilde{H}_{\bar{r}}\right)$ is satisfied. Take any nontrivial interval $[s, t] \subset[S, T]$ such that $\mathcal{N}_{[s, t]}=\bar{r}+1$. We can assume that

$$
h_{j}(s, \bar{x}(s))=0 \quad \text { for all } j \in \mathcal{A}_{[s, t]},
$$

for otherwise there exists some $\tau \in(s, t)$ such that $\mathcal{N}_{[s, \tau]} \leq \bar{r}$. But then, for some nontrivial subinterval $\left[s^{\prime}, t^{\prime}\right] \subset[s, t], \sum_{j} \int_{s^{\prime}}^{t^{\prime}} \mu_{j}(d \sigma) \leq K_{\bar{r}}\left|t^{\prime}-s^{\prime}\right|^{1 / 2}$ by the induction hypothesis. Likewise, we can assume that

$$
h_{j}(t, \bar{x}(t))=0 \quad \text { for all } j \in \mathcal{A}_{[s, t]},
$$

It follows from (6.39) and (6.40) that

$$
\sum_{j} \int_{s}^{t} \mu_{j}(d \sigma) \leq K^{\prime}|t-s|^{1 / 2}
$$


where $K^{\prime}$ is the constant of Lemma 5.7. We have shown that $\left(\bar{H}_{\bar{r}+1}\right)$ is true with $K_{r \overline{+} 1}=$ $\max \left\{K_{\bar{r}}, K^{\prime}\right\}$. (ii) is confirmed.

Consider the case $r=1$. In view of the preceding lemma, $q(\cdot)$ is Hölder continuous with index $1 / 2$. But $\bar{x}$ is Lipschitz continuous. We also know from Lemma 5.1 that $\nabla_{y} L_{0}^{*}(t, x, y)$ is Hölder continuous in $t$ and Lipschitz continuous in $(x, y)$. It follows from these facts and representation (6.38) that $\bar{u}$ is Hölder continuous with index $1 / 2$.

Consider finally the case $r>1$. Take any interval $[s, t]$. We know that there exists $k_{1}$, not depending on $[s, t]$, such that

$$
|q(t)-q(s)| \leq k_{1}\left(|t-s|+\sum_{j} \int_{s}^{t} \mu_{j}(d s)\right) .
$$

It follows from (6.38) and Lemma 5.1 however that, for some $k_{2}$, not depending on $[s, t]$,

$$
|\bar{u}(t)-\bar{u}(s)| \leq k_{2}\left(|t-s|^{1 / 2}+\sum_{j} \int_{s}^{t} \mu_{j}(d s)\right) .
$$

We conclude from Lemma 6.2 existence of $k_{3}$ such that, given any nontrivial interval $[s, t]$, there exists a nontrivial subinterval $\left[s^{\prime}, t^{\prime}\right] \subset[S, T]$ such that

$$
\left|\bar{u}\left(t^{\prime}\right)-\bar{u}\left(s^{\prime}\right)\right| \leq k_{3}\left|t^{\prime}-s^{\prime}\right|^{1 / 2} .
$$

The remaining assertions of Thm. 4.3(A) are a simple consequence of this property. Proof of Thm. 4.3 is complete.

\section{References}

[1] J.F. Bonnans and A. Shapiro. Perturbation Analysis of Optimization Problems. SpringerVerlag, New York, 2000.

[2] A. L. Dontchev. Perturbations, Approximations and Sensitivity Analysis of Optimal Control Systems, Lecture Notes in Control and Information Sciences, Springer-Verlag, Berlin, 1983.

[3] A.L. Dontchev and W.W. Hager. A new approach to Lipschitz continuity in state constrained optimal control. Systems and Control Letters, 35:137-143, 1998.

[4] A.L. Dontchev and I. Kolmanovsky. On regularity of optimal control. Recent Developments in Optimization Proc. French-German Conf. on Optimization, Eds. R. Durier, C. Michelot, Lecture Notes in Economics and Mathematical Systems, 429:125-135, 1995.

[5] G.N. Galbraith and R.B. Vinter. Lipschitz Continuiuty of Optimal Controls for State Constrained Problems, SIAM J. Control and Optimization, 42:1727-1744, 2003.

[6] W.W. Hager. Lipschitz continuity for constrained processes. SIAM J. Control and Optim., 17:321-338, 1979.

[7] K.M. Malanowski. On the regularity of solutions to optimal control problems for systems linear with respect to control variable. Arch. Auto. i Telemech., 23:227-241, 1978.

[8] B.S. Mordukhovich. Generalized Differential-Calculus for Nonsmooth and Set-valued Mappings. J. of Mathematical Ananlysis and Applications, 183 (1): 250-288, 1994. 
[9] S.M. Robinson, Strongly regular generalized equations. Mathematics of Operations Research, 5: 43-62, 1980.

[10] R.T. Rockafellar and R. J-B. Wets. Variational Analysis. Springer-Verlag, Berlin, 1998.

[11] R. B. Vinter. Optimal Control. Birkhäuser, Boston, 2000. 